\title{
Populisme d'extrême droite en France: une analyse de la communication non verbale de Marine Le Pen
}

Morgane Belhadi

Université Sorbonne Nouvelle, Paris 3

\section{Introduction}

De I980 à nos jours, le Front National a complètement changé sa stratégie communicationnelle, désormais incarnée par Marine Le Pen. Cette dernière, qui a remplacé en 20 I I son père à la tête du FN avec succès, entend modifier l'image du parti d'extrême droite pour le rendre plus acceptable.

Cet article a pour objet l'évolution de l'image de Marine Le Pen à travers l'analyse de deux débats : le premier datant de 2002 l'oppose à Julien Dray suite à l'accession de Jean-Marie Le Pen au second tour de l'élection présidentielle pour la première fois de l'histoire du parti, le second débat confronte la désormais candidate aux présidentielles de 20I 2 à Jean-Luc Mélenchon, lequel est souvent qualifié de populiste d'extrême gauche.

Cette étude sera conduite par le biais de la communication non verbale, ćest-à-dire la manière dont les individus se présentent en termes de gestuelle, de posture, d'expressions faciales et de tenue vestimentaire. En effet, malgré sa complexité d'analyse et sa polysémie, la communication non verbale joue un rôle non négligeable dans la mise en scène, la constitution de l'ethos et de l'image publique des responsables politiques et leur processus de persuasion et, à plus forte raison, auprès des leaders populistes, qui fondent leur rhétorique sur des images et phrases frappantes.

Comment la communication non verbale s'est-elle développée dans le champ de la communication politique ? Que dit-elle de la stratégie

Comment citer ce chapitre :

Belhadi, M. 2019. "Populisme d'extrême droite en France : une analyse de la communication non verbale de Marine Le Pen ». In: Françoise Sullet-Nylander, María Bernal, Christophe Premat \& Malin Roitman (eds.). Political Discourses at the Extremes. Expressions of Populism in Romance-Speaking Countries. Stockholm Studies in Romance Languages. Stockholm: Stockholm University Press, pp. I49-I74. DOI: https://doi.org/Io.I6993/bax.h. License: CC-BY 
politique de Marine Le Pen en tant que représentante d'un parti souvent qualifié de populiste et d'extrême droite ? Pour mieux le comprendre, nous explorerons les différents apports théoriques sur le non verbal en politique, avant d'analyser un corpus audiovisuel qui permettra de vérifier son influence croissante et, in fine, de mieux saisir le virage discursif opéré par le FN, devenu Rassemblement national (RN) depuis le $\mathrm{I}^{\text {er }}$ juin $20 \mathrm{I} 8$.

\section{Communication non verbale et communication politique : éléments de définition et apports disciplinaires}

La communication non verbale renvoie à l'image que l'on donne de soi à travers l'apparence physique. Dans une acception plus étroite, elle concerne les éléments visuels physiques susceptibles d'ajouter, de compléter ou de contredire le sens de l'énoncé émis. Elle a ainsi pour objet l'analyse de la " posturo-mimo-gestualité » (Cosnier I984), dimension inhérente au physique d'un individu et au message qu'il porte.

Expressive, dialogique, performative et symbolique, elle fait plus qu'accompagner la parole et, loin d'aller de soi, elle est à la fois psychologiquement, socialement et culturellement construite. En outre, l'histoire de la politique ne peut se penser sans sa portée symbolique qui se mesure à des mots, mais aussi à un style, une attitude, des gestes et des images mémorables. Bien avant l'essor des médias, le politique a toujours été en représentation (Abélès 2007).

$\mathrm{Au} \mathrm{XX}^{\mathrm{e}}$ siècle, le psychologue américain Paul Ekman (I975, I976) reprend l'idée darwinienne de l'universalité des émotions et en dénombre sept - la haine, la colère, le dégoût, la peur, la joie, la tristesse et la surprise - qui enclenchent selon lui les mêmes mouvements musculaires du visage pour tous les individus.

Le psycholinguiste David McNeill (I992), quant à lui, persuadé que la parole ne peut se penser sans la gestuelle en tant qu'expression des pensées de l'individu, conduit une expérience sur plusieurs sujets aux profils variés qui doivent décrire l'extrait d'un dessin animé qu'ils ont visionné : chacun décrit la même scène en des termes similaires mais avec des gestes différents, selon leurs préférences personnelles, mais aussi en fonction des moments de la scène qu'ils estiment les plus marquants ou pertinents. Il identifie enfin ce qu'il appelle le "growth point ", soit la synchronie, la simultanéité et l'unité entre le discours, la pensée et le geste du locuteur.

Adoptant un angle plus large, anthropologues et sociologues décident, pour leur part, d'explorer le non verbal par le prisme du contexte 
socio-culturel, à l'instar du sociologue canadien Erving Goffman. Celui-ci décrit de manière édifiante le fonctionnement des interactions sociales dans les situations les plus ordinaires, les rites, règles et normes implicites et explicites qui y sont à l'œuvre (l'« ordre » de l'interaction). "Par interaction (c'est-à-dire l'interaction face à face), on entend à peu près l'influence réciproque que les partenaires exercent sur leurs actions respectives lorsqu'ils sont en présence physique immédiate les uns des autres»(Goffman I973:44). Ainsi, chaque acteur - au sens dramaturgique du terme - de l'interaction ajuste son comportement en fonction de l'interprétation qu'il tire de la situation, de l'image qu'il se fait de l'autre, et se constitue une "face ", c'est-à-dire une identité publique. Pour ce faire, il travaille sa "façade ", soit tout l' " appareillage symbolique " mobilisé pour instaurer sa propre représentation (décor, apparence physique, gestuelle, etc.). Cette «face » est plus ou moins maîtrisée, cohérente, maintenue, car certaines informations - provenant généralement de l'incongruence entre ce que le corps manifeste et ce que le verbal exprime - peuvent échapper à la vigilance de l'acteur ou relever de son inconscient.

À la suite de Goffman, nombre d'auteurs vont travailler sur plusieurs moments de communication, dans une approche interactionnelle parfois doublée d'une approche multimodale. Dans le domaine de la linguistique, Patrick Charaudeau (I995) observe que les interactants établissent ce qu'il appelle un " contrat de communication " au cours duquel ils opèrent trois stratégies discursives : la légitimation, la crédibilité et la captation (séduire, captiver, toucher l'affect, susciter l'émotion du destinataire). Or, nous verrons que notre corpus audiovisuel rend clairement compte du « choc » des ethos, des luttes pour la reconnaissance et l'existence de chaque débatteur (Charaudeau I993) et de son ambition d'occuper l'espace médiatique en conjuguant ces trois stratégies discursives de la manière la plus satisfaisante possible tout en cherchant à dévaloriser le concurrent (Poggi, D'Errico, Vincze 20I I).

Toujours dans le champ de la linguistique, alors que dans les années I950 l'anthropologue Ray Birdwhistell (Winkin I984) présentait la kinésique, modèle complexe inspiré de la linguistique descriptive, mais difficilement applicable, Geneviève Calbris (2003) propose une méthode d'analyse générale des gestes coverbaux - expression qu'elle reprend du psychologue Jacques Cosnier (I984). Elle va s'en servir pour étudier la gestuelle de Lionel Jospin, dans une approche sémio-linguistique qui prend en considération les travaux antérieurs sur le non verbal, notamment ceux de David McNeill. Elle ouvre ainsi 
la voie pour une recherche sur la communication non verbale des acteurs politiques.

Ce bref panorama permet de mieux saisir le rôle accordé, directement ou indirectement, à la communication non verbale dans la sphère scientifique, qu'il s'agisse, selon la préoccupation et l'approche des auteurs, de mieux comprendre les comportements humains et relations sociales, ou de décrypter le langage multimodal des politiques. Voyons à présent comment ces méthodes s'illustrent, à travers l'analyse d'un corpus audiovisuel.

\section{La communication non verbale en action : analyse de l'ethos de Marine Le Pen}

\subsection{Choix du corpus audiovisuel et questionnements}

Le débat télévisé, "mode fondamental d'expression et d'élaboration du politique " (Jacquin 20I I : 45), semble le mieux à même de rendre compte de la mise en confrontation des idées, la plupart du temps antagoniques, de deux ou plusieurs politiques et de leur manière d'interagir et de défendre leur ethos. En effet, le débat suppose nécessairement une confrontation, soit l'" opposition d'au moins deux points de vue sans pour autant que les énonciateurs qui les prennent à leur charge ne s'orientent vers la possibilité d'un accord [...], la présence d'un tiers garantissant la présence et le maintien du désaccord » (Jacquin 20I I : 45), encourageant éventuellement la polémique pour rendre le débat plus attractif et spectaculaire. Si point de polémique n'est créée dans nos deux extraits, la confrontation et la scénographie qui l'accompagne provoquent néanmoins une tension "dramatique " forte, pour filer la métaphore théâtrale, qui va grandement influer sur les comportements - verbaux et non verbaux - des participants : dans les deux séquences audiovisuelles, Marine Le Pen suscite chez ses deux contradicteurs la colère - involontairement semble-t-il, concernant Julien Dray qu'elle tente d'apaiser - et leur détermination à affaiblir l'ethos de celle-ci.

Ainsi, comme l'ont bien souligné à leur manière Charaudeau et Goffman, au cours de l'interaction, le but de chaque débatteur est double : affirmer son identité par plusieurs techniques et stratégies discursives, la "co-construire " (Constantin de Chanay et Turbide 20I I) selon les réactions du camp adverse et de la réception, et, par contraste, décrédibiliser l'autre, lui faire "perdre la face ».

Mais la félicité du débat et de l'interaction ne peut être assurée qu'en conformité à des règles, normes et attentes spécifiques connues 
et acceptées de toutes les instances - politique, médiatique et citoyenne (Charaudeau 2002) pour le bon fonctionnement de l'émission - prises de parole contrôlées, thèmes imposés, courtoisie, clarté des propos tenus - dont le modérateur se fait le garant.

\subsection{Méthodologie de l'analyse}

Trois auteurs principaux ont retenu notre attention : Geneviève Calbris (2003), David McNeill (I992) et Paul Ekman (1975, I976), dont les concepts qui semblaient les plus pertinents ont été adaptés aux extraits choisis. Quatre éléments sont considérés : la tenue vestimentaire, la scénographie filmique, et surtout les expressions faciales et les gestes coverbaux. Est écartée de l'analyse la prosodie que l'on range dans la catégorie paraverbale.

Les gestes coverbaux peuvent remplir différentes fonctions : une fonction redondante, de substitution de la parole et que Ekman nomme les « emblèmes ", une fonction de complément (ce sont les "illustrants »), ou bien la manifestation d'une gêne, par exemple, dans le cas de gestes d'auto-contact de démangeaison (qu'on appellera les «manipulatoires »).

Ils sont l'expression de plusieurs degrés d'abstraction du discours (McNeill I992) : les "iconic", les "metaphoric", les "deictic" et les "beats ", quatre gestes qui peuvent se superposer et sont répartis différemment dans l'espace, à proximité du locuteur ( "center-center») ou éloignés ( extreme periphery "). Les "iconic " font référence à " un événement concret, un objet, ou une action auquel le discours se réfère simultanément " (McNeill I992); les "metaphoric " créent des images abstraites donc entretiennent un lien analogique et symbolique avec ce qui est dit ; les "deictic » permettent de désigner quelque chose ; et les «beats» se traduisent souvent par de petits mouvements des mains ou de la tête et rythment le discours ou la conversation.

Inspirée de la sémiologie et de la psycholinguistique, avec en toile de fond la communication politique, la méthode repose sur plusieurs étapes : isoler les gestes et les mouvements faciaux les plus récurrents ou significatifs dans un premier temps, puis les resituer dans la totalité du mouvement effectué et la situation d'énonciation afin d'éviter l'écueil de la description et de l'isolement du non verbal du contexte.

Nous donnons au contexte un sens élargi, en tant qu'il concerne plusieurs canaux de communication :

- la configuration physique du plateau ou «dramaturgie proxémique » (Jacquin 20I I : 49) : dans les extraits étudiés, un voire 
deux journalistes modérateurs dominent une table qui sépare les deux invités politiques, le plateau est de taille moyenne, et le public situé en arrière-plan pour renforcer l'intimité et la dimension spectaculaire et interactionnelle de l'émission;

- le contexte visuel filmique : dans les extraits étudiés, les plans les plus fréquents sont le plan d'ensemble et le plan rapproché qui cadre les participants jusqu'aux épaules, l'usage du split screen et du gros plan qui ne montre que le visage pour renforcer la confrontation et la dramatisation du débat ;

- les composantes physiques du mouvement corporel étudié : la main, le regard, la bouche, le bras, le buste, plusieurs de ces éléments à la fois, son orientation, sa séquentialité (sa rythmique et son apogée), comprendre que le mouvement corporel a souvent un effet d'annonce, qu'il précède la parole et est lui-même souvent annoncé par le regard;

- le contexte politique, médiatique et la situation d'énonciation : sujet sur lequel porte l'extrait, liens entre le verbal et le non verbal, mode de diffusion - ici en direct - et enfin statuts et positionnements de l'instance télévisuelle, qui, par ses choix scénographiques, journalistiques, idéologiques et économiques et son intentionnalité, conditionne "le regard spectatoriel » (Esquenazi I999 : г 8) en tentant d'identifier les attentes du téléspectateur «lambda»;

- la réception : comment le débat a-t-il été reçu, compris ?

Cette méthodologie s'achève par les inévitables limites pratiques posées à l'analyse. En effet, il est difficile de repérer les micro-expressions identifiées par Ekman comme les mimiques du visage qui durent moins d'une seconde et sont quasiment invisibles à l'œil nu. Les techniques de filmage privilégient les cadrages resserrés, lesquels tronquent souvent les gestes effectués par les mains, qui sont pourtant très souvent sollicitées. De plus, les techniques de cadrage peuvent influencer de manière positive ou négative le " regard spectatoriel " sur le débat et ainsi renforcer ou affaiblir l'ethos du politique, d'où la grande susceptibilité des candidats et de leurs équipes au sujet des modalités de mise en scène des débats présidentiels d'entre-deux-tours en particulier. Enfin, le format bidimensionnel de l'écran télévisé produit un écart sur la perception visuelle et la réception, par rapport à la réalité, qui, elle, est tridimensionnelle. 


\subsection{Extrait de Mots croisés, émission spéciale sur " La montée du populisme ", présentée par Arlette Chabot, France 2, 27.05.2002}

Notre corpus audiovisuel a été conçu pour vérifier l'évolution entre 2002 et 2012 de la dimension non verbale de Marine Le Pen dans un contexte de montée spectaculaire des populismes en Europe, mais aussi afin d'examiner les interrogations suivantes : comment se construit l'interaction, y compris lorsqu'elle n'est pas voulue (refus de débattre de Le Pen dans le deuxième extrait) ? En quoi la communication non verbale peut constituer une ressource non négligeable, particulièrement s'agissant du deuxième débat ? Enfin, quels sont les apports de la communication non verbale à la compréhension de ces deux extraits et plus généralement à l'analyse interactionnelle et multimodale d'un débat politique télévisé ?

Le premier extrait, dont l'intégralité peut être visionné aux archives de l'Institut National de l'Audiovisuel (InaTHÈQUE) à la Bibliothèque nationale de France à Paris, est issu de Mots croisés, émission diffusée sur France 2, qui apparaît pour la première fois en I997, après le Journal télévisé de $20 \mathrm{~h}$. A partir de $200 \mathrm{I}$, elle est programmée en deuxième partie de soirée, suite à la création de l'émission Complément d'enquête qui commence à 20 h 50 . L'émission qui nous intéresse est consacrée au populisme. Y cohabitent des invités issus de milieux différents (intellectuels, journalistes et politiques). Après plusieurs discussions et reportages sur le sujet, arrive le débat, animé par Arlette Chabot, entourée de plusieurs intervenants : Marine Le Pen, Roselyne Bachelot, Julien Dray, Olivier Besancenot, Dominique Wolton et George-Marc Benamou, écrivain-journaliste. La séquence que l'on va étudier confronte Julien Dray à Marine Le Pen et dure trois minutes.

Le débat s'ouvre sur un Julien Dray tendu : ses sourcils sont froncés, ce qui provoque des rides verticales, son regard est fixe, ses lèvres pressées l'une contre l'autre, et son visage ruisselle. Il semble déterminé à démonter l'argumentation de Marine Le Pen, ce qui n'a pas échappé à la principale intéressée. Mais plutôt que de se montrer offensive, en accord avec sa stature imposante qui rappelle celle bien connue de son père, Marine Le Pen tente une stratégie de la séduction dans le but de l'apaiser. Calme et à l'écoute, ses gestes sont ouverts vers l'extérieur (vers la "périphérie extrême " pour reprendre David McNeill), la tête en avant en direction de son contradicteur. Elle opère également un mouvement de balancier du buste de l'arrière vers l'avant comme si elle s'avançait vers lui pour mieux le convaincre. Enfin, elle lui adresse de 
fréquents regards, pour l'impliquer plus fortement. Or, l'on sait combien le regard joue un rôle important dans l'interaction (Constantin de Chanay, Kerbrat-Orecchioni 20I7) : selon qu'il est orienté vers le délocuté ou le modérateur-journaliste, furtif ou insistant, incluant le délocuté ou l'excluant - ici, manifestement, il est un incluant et appuyé à l'intention de Dray -, il n'aura pas la même incidence sur l'interprétation de la situation d'énonciation et la structuration des ethos des deux débatteurs.

Julien Dray possède alors un capital politique et une visibilité médiatique plutôt faibles et est critiqué par son train de vie. Mais son expérience, son appartenance au Parti socialiste et son implication dans la fondation de SOS Racisme font de lui un redoutable opposant aux idées conservatrices du Front National, accusé, entre autres, de populisme et de racisme.

L'enjeu de "dédiabolisation » est donc de taille pour Marine Le Pen, qui, afin de se légitimer tout en se victimisant, n'hésite pas à déplorer : "J'en ai marre d'être traitée de raciste, je ne suis pas raciste ! ". Le passage du "nous ", employé antérieurement, relatif au parti frontiste, au « je », lui permet de s'impliquer personnellement et de se mettre en avant. Elle adresse également des regards successifs aux autres invités ainsi qu'à Arlette Chabot, afin de les impliquer personnellement, voire, peut-être, de les accuser eux aussi de l'avoir traitée de " raciste » par le passé. Cette stratégie semble être appuyée par les mouvements de caméra avec un gros plan sur son profil, un plan sur Julien Dray puis sur Olivier Besancenot, lesquels ont respectivement les yeux baissés, comme s'ils se sentaient visés par ses propos.

Néanmoins, Marine Le Pen ne parvient pas complètement à masquer son malaise : elle tente par exemple d'interrompre Julien Dray, avant de consentir sans grande résistance à lui céder la parole, et elle cligne des yeux très souvent - au calme, une personne normale cligne des yeux en moyenne toutes les cinq secondes (Mery 20I4) -, signe de stress.

Si nous visionnons l'émission plus loin, le contraste avec Olivier Besancenot est saisissant. Sans utiliser le moindre mot, simplement à travers son visage, Marine Le Pen lui exprime tout son dédain et sa condescendance : elle soupire, souffle, détourne le regard pour feindre le désintérêt et l'ennui face au discours de Besancenot, mais aussi pour créer une sorte de connivence avec les autres invités dont on peut entendre les objections en hors champ pendant que Besancenot s'exprime. On peut donc ici parler de délocution excluante ; l'objectif est de montrer qu'elle ne le reconnaît pas comme contradicteur sérieux, malgré 
la forte progression qu'il a réalisée dans les suffrages exprimés en 2002 $(4,25 \%$ au lieu des $0,5 \%$ prédits), mais loin derrière Jean-Marie Le Pen ( $17,79 \%$ des voix au second tour). La différence d'ethos est donc significative. Olivier Besancenot exerce une profession de facteur, bien loin du monde politique, il se positionne en outsider, à l'extrême gauche (il est alors membre du parti LCR, la Ligue Communiste Révolutionnaire) et emploie un registre de langue courant voire familier, fondé sur le pathos, l'appel à l'émotion et à l'indignation. À l'inverse, Julien Dray, malgré sa colère et sa détermination manifestes, construit son argumentation sur des idées rationnelles, le logos. Marine Le Pen n'est donc pas intimidée par Olivier Besancenot.

Voyons à présent quelques traits non verbaux marquants de Marine Le Pen qui se manifestent au cours de cet extrait :

Ses gestes sont organisés : lorsqu'elle dit « «Je vous ai compris». Alors on nous a fait le coup en 88, 95, et on nous fait le coup en 2002 ", ses mains se répartissent en fonction de la date évoquée (à gauche, à droite, au centre) afin de renforcer son propos et de le rendre d'autant plus intelligible et concret : on retrouve ici la fonction des gestes " iconic » identifiés par David McNeill (I992).

On reconnaît également un geste que Calbris (2003) avait repéré chez Lionel Jospin : la main en pointeau dirigée vers le ciel, qu'elle qualifiait de "Pyramide ». Le Pen en fait la même utilisation que Jospin, c'est-à-dire pour préciser sa pensée, mettre en avant un élément de son discours qui lui paraît essentiel : "Or cette souffrance [éprouvée par les Français], vous l'avez méprisée, vous l'avez bafouée ». Ce geste fonctionne en congruence avec sa parole puisque, sur le plan verbal, elle utilise le démonstratif « cette » et le pronom personnel «l' » qui remplace la «souffrance ", tout en insistant sur la première syllabe des verbes "mépriser » et "bafouer ». Cette construction grammaticale, corroborée par le geste de la Pyramide dont les doigts sont dirigés vers le haut, semblent indiquer la "souffrance » et servent ainsi sa rhétorique, qui consiste à mettre en avant le peuple et à montrer son empathie pour lui. L'adresse directe à un "vous » abstrait - mais l'on peut supposer qu'il s'agit du système politico-médiatique et des partis de gouvernement dont Le Pen souhaite s'extraire - suggère que seul le Front National serait capable d'écouter la "souffrance » du peuple et d'y mettre fin.

De même, lorsqu'elle déplore que ne soient pas évoqués certains sujets sensibles ("Quid de l'immigration, quid de l'Europe, quid de la corruption »), elle réitère le geste de la Pyramide qu'elle agite ( "beat », McNeill I992) à chaque fois qu'elle prononce le mot « quid » pour scander son 
propos aux plans verbal et non verbal et créer un effet d'insistance et d'accumulation. Elle poursuit : "Si c'est si simpliste que cela ce que propose le Front national, eh bien vous allez facilement faire comprendre aux Français qu'ils ont tort » en effectuant le geste de ce que Calbris (2003) appelle le "Livre entrouvert ", qui se caractérise par le fait d'ouvrir les mains, paumes dirigées vers le haut. Aussi qualifié de «Palm Up Open Hand » (PUOH) par McNeill (I992), ce geste métaphorique permet à Marine Le Pen de souligner avec ironie la fausse " simplicité » et « facilité » - élément abstrait, d'où le rôle joué par le geste métaphorique avec laquelle ses adversaires vont « faire comprendre aux Français qu'ils ont tore [d'adhérer aux idées du Front national] ».

Enfin, un élément caractéristique de la personnalité de Le Pen et de sa rhétorique populiste est sans doute son ironie, qui lui permet d'instaurer une distance et de creuser le clivage ${ }^{\mathrm{I}}$ par rapport à son adversaire tout en le dévalorisant. Caractéristique essentiellement verbale, l'ironie trouve ici son corollaire sur le plan non verbal. En effet, Le Pen fronce fréquemment les sourcils pour imiter les propos de ses concurrents et mettre en doute leur cohérence. Au moment où Julien Dray affirme que le Parti socialiste est favorable à une part de proportionnelle, elle esquisse un faux sourire, qui fonctionne comme une antiphrase (Kerbrat-Orecchioni 20I3 : 52) des propos tenus par Dray : si les commissures des lèvres sont bien élevées comme dans les vrais sourires, il n'y a pas de patte d'oie qui se dessine.

\subsection{Extrait de l'émission Des paroles et des actes, présentée par David Pujadas, France 2, 23.02.2012}

Malgré le caractère technique et pointu qu'elle revêt, Des paroles et des actes, née en $20 \mathrm{II}$, opère une sorte de synthèse des genres télévisés. Cette émission conserve un caractère éminemment sérieux dans la pure tradition des émissions politiques, avec des aspects spectaculaires, comme c'est le cas des émissions d'infodivertissement. Il s'agit notamment des modes de filmage, tels que le split screen, qui renforcent la dramatisation et la mise en confrontation, des temps de parole limités comme dans les débats présidentiels, des sondages révélés à la fin, des questions du public, et de l'évaluation des prestations des invités par les journalistes ; on note aussi des nouveautés pour mieux servir l'intérêt démocratique et informationnel du téléspectateur (le fact-checking).

\footnotetext{
${ }^{\text {I }}$ Rabatel qualifie d'ailleurs l'ironie de « raillerie clivante qui oppose Moi/Nous [...]
} vs. Eux ", comme le rappelle Kerbrat-Orecchioni (2013 : 49). 
A dix ans d'intervalle - 2002 et 2012 -, le changement d'apparence de Marine Le Pen est manifeste. Ce changement participe clairement d'un travail sur son " appareillage symbolique » pour satisfaire aux exigences du parti alors en pleine rénovation : un brushing parfaitement maîtrisé (cheveux coupés mi-longs, couleur blonde éclaircie), une importante perte de poids, des tenues et un maquillage plus élégants et discrets attestent de sa volonté de s'afficher plus moderne et dynamique, sans pour autant renoncer à des éléments de continuité de l'idéologie frontiste pour préserver le noyau dur de son électorat : la blondeur, courante dans les partis d'extrême droite européens, une chaîne en or ornée d'une croix chrétienne, une tenue un peu grande pour elle qui renforce sa stature imposante, probablement en écho à celle de son père.

Ce changement de look est survenu en 2004, au moment des élections européennes et à l'approche du référendum de 2005 sur la Constitution européenne. De plus, Marine Le Pen a changé de statut en 20 I I, accédant à la présidence du Front National, et en 20I2, elle se présente pour la première fois à une élection présidentielle. Le travail sur son apparence est donc concomitant de son entreprise de conquête du pouvoir à l'échelle nationale et européenne et l'émission que nous choisissons de traiter représente donc un enjeu pour elle.

À l'occasion de l'élection présidentielle de 2012, Des paroles et des actes a consacré chacune de ses émissions à un candidat, dont celle du 23 février à Marine Le Pen. L'émission est composée de trois parties : dans la première et la dernière, des journalistes posent des questions à l'invité sur son programme économique, politique et social, sur son image puis sa prestation; la deuxième partie est un duel opposant deux politiques. Habituellement d'une durée de quarante minutes, il s'agit ici de deux duels de vingt minutes chacun - l'un face à Henri Guaino, représentant de Nicolas Sarkozy, l'autre à Jean-Luc Mélenchon, alors président du Front de gauche. L'imposition de deux duels plutôt qu'un fait suite à la demande de la frontiste qui souhaitait être confrontée à un représentant de son "niveau électoral ", les sondages plaçant Mélenchon derrière elle à la présidentielle.

Le deuxième duel, que nous allons donc analyser et qu'il est possible de visionner en entier sur le site de vidéos en ligne YouTube, donne à voir deux candidats issus de partis extrêmes qualifiés de populistes. Avant même de connaître l'issue du débat, l'horizon d'attente du téléspectateur d'assister à une émission spectaculaire et à rebondissements, se trouve fortement conditionné, compte tenu des rapports antérieurs plutôt houleux entre les deux débatteurs, qui ont multiplié les attaques ad hominem par médias interposés. 
A l'entrée de Mélenchon sur le plateau, la tension s'installe donc rapidement : celui-ci affiche d'emblée sa détermination et sa concentration sur les sujets qu'il a choisi d'aborder, comme l'attestent l'expression de son visage - froncement des sourcils, index accusateur ("deictic»), regard fixe et déterminé, beaucoup de mouvements de main en direction de Marine Le Pen pour l'impliquer et l'accuser - et le contenu de son propos. "Ce qui nous séparera nos deux courants ", phrase incorrecte grammaticalement, est toutefois révélatrice de sa pensée, soulignant combien tous deux s'opposent aussi bien sur le plan des idées (" nos deux courants ») que des personnalités (" nous séparera »). Cette dualité se retrouve dans la scénographie de l'émission qui renforce la dramatisation du débat, en cohérence avec l'horizon d'attente du public : on trouve plusieurs split screen séparant Mélenchon à droite et Le Pen à gauche, plans rapprochés et zooms sur les visages pour donner à voir les réactions de chacun et accentuer le suspense.

Comme dans l'extrait de 2002, l'ironie de Le Pen passe par le mime pour dévaloriser son adversaire : "Vous êtes un peu le Paul Amar des temps modernes, dit-elle à David Pujadas. Vous auriez pu nous donner, peut-être, une paire de gants de boxe [en référence au débat opposant Jean-Marie Le Pen et Bernard Tapie en I994 au cours duquel le présentateur, Paul Amar, leur avait fourni une paire de gants de boxe]. D'ailleurs, Mélenchon a tout de suite parlé de combat de boxe, de me traîner, je crois, d'un bout à l'autre du ring avec ses dents peut-être ". Ses derniers mots s'accompagnent de gestes et de mouvement de balancier de son buste pour mimer le combat sur le ring, face à un Mélenchon sourcils froncés, interloqué. Or, cette référence au débat de son père et au monde de la boxe, fondée sur le conflit physique et non sur le conflit des idées, visent pour Marine Le Pen à dévaloriser son adversaire et à montrer que le statut de victime de son parti n'est pas récent (I994). Ce moment participe de la dramatisation du duel, servant les ambitions de la chaîne de rendre le débat attractif, et pas seulement technique. Là encore, les rapports entre télévision et politique sont à considérer si l'on veut comprendre le contexte dans lequel s'inscrit l'émission.

Ironie et victimisation se doublent d'une stratégie de l'évitement, qui n'est pas sans lien avec le fait que Marine Le Pen est créditée de plus de voix dans les sondages que Jean-Luc Mélenchon, ce qui la met en position de force et lui permet d'afficher une attitude condescendante à l'égard de celui-ci. L'usage du verre d'eau à plusieurs reprises au cours de l'extrait, le refus de regarder Mélenchon, le fait de feindre de lire des titres disposés sur sa table - qui ne sont autres que La Voix du Nord, 
quotidien lié à une région dans laquelle l'implantation du FN s'accroît, et Le Point titrant "Qui ment le plus ? » entre les candidats de droite et de gauche - sont autant d'indicateurs non verbaux et visuels qui soulignent le mépris et le désintérêt affichés par Le Pen.

Comme le rappelle Marlène Coulomb-Gully, « la politique à la télévision, c'est d'abord de la télévision » (200 I : I I) : ici, l'émission semble soutenir cette stratégie, quoique involontairement peut-être, à travers ses différents choix de filmage. Plutôt que de filmer Mélenchon, qui déploie une argumentation méthodique pour dénoncer le programme du FN, la caméra montre les réactions d'ennui de Le Pen lorsqu'elle fait mine de réorganiser ses papiers. Sa volonté de faire diversion et de dévaloriser la parole de son adversaire se révèle payante puisque c'est ce que l'on voit à l'écran. De plus, elle est la seule des deux invités à bénéficier d'un quasi très gros plan (son front et son menton sont tronqués) au cours de l'émission. Enfin, dans un plan, la caméra crée une illusion optique, donnant l'impression que Le Pen est au sommet d'un triangle imaginaire dont Mélenchon et Pujadas seraient la base, alors qu'en réalité, c'est Pujadas, en arbitre du débat, qui domine l'espace. Le mode de filmage et la "dramaturgie proxémique " construisent donc un « regard spectatoriel » qui ne correspond pas nécessairement à la configuration réelle du plateau et qui peut influencer le téléspectateur dans son jugement du débat.

Or, malgré une stratégie bien affûtée, Le Pen se sent-elle si à l'aise dans cette posture ? On note quelques signes de nervosité et de stress : elle cligne beaucoup des yeux, cherche à faire diversion du sujet que Mélenchon tente d'imposer et le regarde très peu (ce qu'il lui reproche d'ailleurs à plusieurs reprises), lui préférant Pujadas situé à sa droite, sans doute pour créer un effet excluant de Mélenchon et une coalition avec le modérateur-journaliste. De plus, elle regarde dans le vide à sa gauche, passe sa main dans ses cheveux, alors qu'ils n'obstruaient pas son champ de vision, elle effectue des mouvements de balancier sur sa chaise qui rendent sa position instable et agitée. On note aussi qu'elle ravale sa salive lorsque Mélenchon arrive et se gratte le nez pendant qu'il déclare par exemple : "Si j'étais un leurre vous n'auriez pas si peur ", ou bien elle garde les bras croisés pendant une bonne partie de l'argumentation de Mélenchon, ce que l'on peut interpréter comme un mécanisme de défense et de rejet à l'égard de son contradicteur. L'objectif de ce dernier est de déclencher la colère de la frontiste, mais elle se contrôle, afin d'assurer sa domination sur lui et sur l'ensemble du débat : "L'émission m'est consacrée », disait-elle au début du duel. 
En outre, si sa première intervention où elle exprimait son refus de débattre était préparée, il semble que les suivantes le soient moins. Au plan verbal, on note beaucoup d'hésitations, de " euh » et de pauses, ce qui n'était pas le cas dans l'extrait de 2002. Ses gestes métaphoriques donnent des indications vagues sur les concepts qu'elle énonce verbalement. Tel est le cas lorsque ses mains dessinent un cercle aux contours imprécis, tandis qu'elle cite, parmi les « candidats suscités par Nicolas Sarkozy », Jean-Louis Borloo, Christine Boutin ou Frédéric Nihous, qui sont selon elle autant de "leurres ".

Quant à Mélenchon, malgré ses sourires ironiques et ses traits d'humour pour maintenir la "face », sa détermination initiale et sa virulence se couplent d'un visage exprimant la colère une fois qu'il a compris que le débat n'aurait pas lieu. A la fin du duel, la colère se double de mépris car il réplique en la regardant avec hauteur, narines dilatées et lèvres pincées : " un cirque [mot employé un peu avant par Le Pen pour qualifier le cadre du débat] dont vous êtes le clown Madame ».

Mais il décide aussi de tirer profit de ce "non-débat " pour démonter le programme du Front National et accroître sa visibilité et son ethos: " votre mépris me laisse indifférent [...] vous ne m'impressionnez d'aucune manière ». Dans ce passage, Marine Le Pen, mal à l'aise, cligne des yeux dix fois à la seconde puis baisse les yeux, avant de les rediriger rapidement vers Mélenchon, pour masquer sa gêne.

Lorsqu'il l'accuse de faire un commerce des mères porteuses avec l'adoption prénatale que son programme préconise, elle feint le doute en fronçant des sourcils, mais cette expression n'accompagne pas de démenti verbal : afin de respecter son objectif de ne pas débattre et de maintenir le rôle (au sens goffmanien du terme) qu'elle s'était assigné, elle s'appuie quasi exclusivement sur sa communication non verbale, au point de ne pas pouvoir défendre ses idées ni de contredire Mélenchon. Son rôle paraît "surjoué ", non conforme aux attentes du public et de l'instance télévisuelle d'assister à un débat classique de confrontation des idées. Sa stratégie montre ici ses limites et fut d'ailleurs plutôt mal perçue par la presse.

\section{Conclusion : apports et limites de la communication non verbale en politique}

La communication non verbale, dimension inhérente à l'expression du politique, a connu une forte expansion dès les années I960 avec la télévision, l'avènement du marketing, et dans les années I980 du 
media-training qui permet aux politiques de s'entraîner pour réussir les prestations médiatiques. Elle est donc bel et bien intégrée à la communication politique. Toutefois, plusieurs questions restent en suspens, comme le fait de savoir s'il existe une communication non verbale propre à chaque idéologie politique, ou relative à une communication plus globale que l'on pourrait trouver dans d'autres domaines que la politique.

Par ailleurs, malgré les propositions de plus en plus nombreuses sur le plan théorique et empirique, il demeure difficile de l'analyser et de mesurer sa réception auprès du public du fait du contexte, du rôle de l'instance télévisuelle et journalistique qui conditionnent l'intervention du politique.

Ensuite, le statut de la communication non verbale pose problème : pas encore discipline scientifique à part entière en France, elle s'inscrit plutôt dans une pratique professionnelle qui ne prend pas encore en charge son contenu théorique : les spécialistes de la communication s'appuient sur la PNL (programmation neuro-linguistique) et la synergologie, deux modèles à la scientificité douteuse, ou sur leur savoirfaire personnel, leurs intermédiaires (acteurs, psychologues, professeurs de chant, entre autres), les envies et les personnalités de leurs clients politiques.

Enfin, elle soulève plusieurs paradoxes. Le politique doit satisfaire l'exigence de vérité, d'honnêteté, paraître naturel... tout en ayant préalablement fourni un travail conséquent et en lissant certains aspects de la réalité. Se pose pour lui deux questions : doit-il privilégier, selon sa personnalité et/ou son idéologie, le pathos avec des gestes pleins d'emphase et de lyrisme... au risque peut-être d'être considéré comme incapable de modération et de dompter ses passions ? Ou à l'inverse, est-il plus astucieux de se concentrer sur la maîtrise de ses émotions et de ses gestes, pour paraître calme et diplomate... mais au risque de l'obsession du contrôle, de donner une image froide et une communication cadenassée ?

Ses effets sont donc à double tranchant lorsque son utilisation est mal pensée, inadaptée aux circonstances ou à la personnalité du politique. Dans le cas de Marine Le Pen, sa communication non verbale devient pour elle un véritable outil stratégique de dédiabolisation, séduction, modernisation et féminisation de son parti, tout en préservant des éléments de continuité historique et filiale avec le parti. Mais cet outil trouve ses limites lorsque dans l'extrait de 20I2, elle choisit de s'inscrire moins dans le logos et l'argumentation comme le fait Mélenchon, que dans le pathos, la victimisation et l'évitement du 
débat en tentant de se désengager de l'interaction. Comme l'écrivait Goffman : "Lorsque des individus se trouvent réunis en des circonstances qui n'exigent pas que des paroles soient échangées, ils s'engagent néanmoins, qu'ils le veuillent ou non, dans une certaine forme de communication [...] Même si un individu peut s'arrêter de parler, il ne peut s'empêcher de communiquer par le langage du corps » (Winkin I984), ce qui rejoint le fameux axiome de Paul Watzlawick : "On ne peut pas ne pas communiquer".

Ainsi, quelle que soit la composante non verbale adoptée, cette étude montre combien il est indispensable au politique d'afficher une posture et une stratégie claires, et une image se voulant la plus naturelle et constante possible pour ne pas laisser penser qu'il est dénué de conviction. Elle révèle également que le travail de Marine Le Pen sur sa communication non verbale s'inscrit dans un changement stratégique plus large visant à transformer le visage du FN (éliminer les éléments perturbateurs, lisser l'image de la candidate pour lui donner l'allure d'une présidentiable, adapter son discours localement), une attitude qui peut s'observer chez d'autres partis populistes européens.

\section{Annexe 1 :}

Tableau récapitulatif des éléments non verbaux effectués par Marine Le Pen, en lien avec le contexte de filmage et la situation d'énonciation (2002). Copyright : Morgane Belhadi. License: CC BY

\begin{tabular}{ll}
\hline Descriptions & Significations \\
\hline $\begin{array}{l}\text { Stature imposante, longs cheveux } \\
\text { blonds }\end{array}$ & Respect des critères de beauté du FN \\
$\begin{array}{l}\text { Gestes ouverts, buste en avant } \\
\text { Julien Dray : regard fixe, sourcils } \\
\text { froncés }\end{array}$ & $\begin{array}{l}\text { Calme et écoute } \\
\text { Colère et détermination }\end{array}$ \\
$\begin{array}{l}\text { Mains de Marine Le Pen disposées à } \\
\text { gauche, à droite, au centre }\end{array}$ & $\begin{array}{l}\text { Organisation temporelle des gestes } \\
: \text { I988 (passé), I995 (passé), 2002 } \\
\text { (présent de l'énonciation) }\end{array}$ \\
$\begin{array}{ll}\text { Main en pointeau (Pyramide) } \\
\text { Caractère essentiel de quelque chose, }\end{array}$ \\
$\begin{array}{l}\text { ici la « douleur éprouvée par les } \\
\text { Flignement d'yeux, détournement » } \\
\text { du regard }\end{array}$ & Gêne face à Julien Dray \\
\end{tabular}




\begin{tabular}{ll}
\hline Descriptions & Significations \\
\hline $\begin{array}{l}\text { Froncement des sourcils, faux } \\
\text { sourires }\end{array}$ & Ironie \\
$\begin{array}{l}\text { Soupirs, détournement du regard, } \\
\text { regards vers les autres invités }\end{array}$ & $\begin{array}{l}\text { Mépris, ennui face à Olivier } \\
\text { Filmage : plans rapprochés, split }\end{array}$ \\
$\begin{array}{l}\text { Renforcement de la dramatisation } \\
\text { du face-à-face, mise en avant des } \\
\end{array}$ & réactions de chacun \\
\hline
\end{tabular}

\section{Annexe 2 : Transcription verbale et non verbale de l'extrait étudié (2002)}

Codifications :

- Entre crochets : indications scéniques et non verbales

- Surligné : mots ou parties de mots qui sont accentués et provoque un ralentissement dans le rythme du discours

- Points de suspension : pauses dans le discours ou moments d'interruption par un autre locuteur

AC (Arlette Chabot s'adressant à Marine Le Pen) Vous, pour vous, vous profitez de quoi ? C'est- à-dire que tous les partis traditionnels selon vous ne parlent pas des sujets ou pas bien des sujets qui intéressent les Français, et vous, vous ramassez tout, quoi, c'est ça ?

MLP (Marine Le Pen) [Ne regarde pas AC, tourne la tête] Ecoutez, le problème n'est pas là. C'est que, les partis traditionnels [visage face caméra, regard dans le vide] comme vous les appelez, parlent de la souffrance du peuple français comme d'un argument [regard vers AC] électoral. Le côté "Je vous ai compris » [regard dans le vide, face caméra], alors on nous a fait le coup [regard vers $\mathrm{AC}$ ] en 88 [mains positionnées à gauche], en 95 [mains positionnées à droite], et on le refait en 2002 [mains positionnées au centre]. Or, cette souffrance [zoom caméra], vous l'avez bafouée [main en pointeau/beat], vous l'avez ridiculisée [main en pointeau/beat], vous l'avez méprisée [main en pointeau/beat]. C’est vous [sourcils froncés imitant les adversaires] qui avez dit des Français qui avaient peur [main en pointeau qui s'ouvre pour désigner la " peur »] de l'insécurité que, euh, c'était un sentiment d'insécurité, que c'était un fantasme, euh, qu'ils étaient repliés sur eux-mêmes [démenti de la tête, sourcils levés]. Ça n'est 
pas vrai ; ils vivaient une situation réelle, et encore aujourd'hui vous les rejetez. Et encore aujourd'hui, vous allez faire en sorte avec vos petites magouilles électorales d'exclure $\underline{20} \%$ des Français d'une représentation à l'Assemblée nationale. Voilà !... [split screen : Julien Dray retire la main de sa bouche, essaye de parler] Voilà la situation...

JD [Julien Dray l'interrompt, sourcils froncés] ... Vous me permettez que je vous pose une question ? Où est la magouille électorale quand on demande une confrontation d'idées ?

MLP La magouille éle... Ah non mais attendez ! [mains dirigées vers JD, se rejoignent] La confrontation des idées, ch'suis parfaitement d'accord avec vous ! Moi [mains d'auto-contact] je suis scandalisée...

JD [interrompt MLP] ... Moi je propose, moi je propose... Moi, j'vais vous dire [MLP essaye de parler] Attendez deux secondes, je finis! Moi, vous savez c'qui m'a posé problème dans l'élection présidentielle ? C’est que y ait ... eu à aucun moment donné [doigt accusateur] ce débat entre les différents candidats en tête à tête ou à deux ou à trois. Et ça, et ça, ça pose un problème [MLP essaye de parler] parce que à ce moment-là, le citoyen effectivement ne peut pas s'y retrouver [regards furtifs alternativement vers AC et vers MLP] et il ne peut pas se faire une idée. Et vous voulez, vous m'parlez d'insécurité ? Ce que j'ai regretté - vous tombez à pic -, j'ai regretté justement, de pas pouvoir avoir un face à face [sourcils froncés] avec un leader de l'opposition ou même [il désigne MLP de la main] avec un leader du Front national sur ces problèmes [MLP hoche la tête de haut en bas] d'insécurité. Moi je l'ai, vous savez, au Conseil régional, avec vos amis [il désigne à nouveau MLP de la main], régulièrement nous débattons de ces questions-là. Je suis pas sûr qu'ils sortent toujours vainqueurs de ce débat.

MLP Ecoutez, Monsieur Dray... [JD essaye de parler] Monsieur Dray, si je puis me permettre de terminer [doigt dirigé vers le haut] [AC essaye de parler] Si je puis me permettre, si je puis me permettre... Le seul problème, le seul problème [poing agité de manière saccadée/beat], c'est qu'aujourd'hui l'insécurité qui vous intéresse, c'est l'insécurité électorale ... [JD soupire, il semble objecter, voix inintelligible] Voilà, quand je parle des magouilles...

JD ... C'est une formule !

MLP Mais non !! Quand je parle des magouilles, c'est que vous êtes en train, vous êtes en train, vous êtes en train de préparer l'élection de 
députés de gauche [mains jointes] ... à, à l'UMP [JD en hors champ : Tant mieux! Tant mieux pour la gauche!] Tant mieux pour vous ! Tant mieux pour vous ! ... Mais est-ce que c'est tant mieux pour les Français ? [JD en hors champ : Pour les salariés] Est-ce que c'est tant mieux pour les Français qui, une nouvelle fois, qui ne vont pas être représentés à l'Assemblée nationale! [JD et AC essayent de parler] Moi, j'suis d'accord avec Monsieur Besancenot [bras désignant $\mathrm{OB}=$ Olivier Besancenot] [plan sur Olivier Besancenot qui l'écoute, impassible]

AC Aah !

MLP ... sur la proposition de la proportionnelle. Voilà ! Pour que l'Assemblée nationale soit enfin représentative de l'intégralité du peuple français [JD essaye de parler] et de sa plus grande part, c'est-à-dire celle du Front national...

JD ... Eh bien, vous voyez, si vous aviez lu le programme du Parti socialiste, nous sommes pour une dose de proportionnelle!

MLP Oui, j'ai appris ça [sourcils froncés], mais vous changez souvent d'avis en fonction de vos intérêts [sourire ironique]

JD Non, non, pas du tout, on change pas d'avis, on pense justement, y compris pour que tous les courants, en créant les conditions de stabilité... [inintelligible]

$[\ldots]$

MLP [...] [s'adressant à Roselyne Bachelot qui accuse le FN et les populismes de proposer des solutions « simplistes »] Le fond du débat ! Quid [main en pointeau/beat] de l'immigration, quid [main en pointeau/beat] de l'Europe, quid [main en pointeau/beat] de la corruption [regard adressé à tous ses interlocuteurs], parlons de, des... des propositions ! Si c'est si simpliste que cela ce que propose le Front national, eh bien vous allez [mains formant un livre entrouvert en direction de ses interlocuteurs] facilement faire comprendre aux Français qu'ils ont tort. Voilà, c'est aussi simple que cela ! On nous fait, aujourd'hui, on se fait traiter de racistes à tout bout de champ ! [sourcils froncés, regard vers $\mathrm{AC}$ ] Moi [regard face caméra] j'en ai marre [sourcils froncés + hochement de la tête de droite à gauche] de m'faire traiter de raciste. Voilà ! Moi je ne suis pas raciste et je ne suis pas xénophobe, je le dis tout net ! [plan sur JD, plan sur OB : ils ont tous les deux les yeux baissés] Et ni moi, ni l'électorat du Front national, ni d'ailleurs les Français 
dans leur ensemble sont ni [gros plan sur le visage de MLP de profil] racistes, ni xénophobes. Ce qu'ils demandent, c'est juste ce qu'ont la majorité des autres pays du monde. C'est les conditions d'accès qu'ont les Etats-Unis, c'est les conditions d'accès qu'ont la Nouvelle-Zélande, alors parlons-en de cela ! Est-ce que c'est honteux, honteux ..., que les Français puissent déterminer qui rentre dans leur pays, quand, pour y faire quoi, combien de temps ? [une spectatrice en arrière-plan de MLP hoche la tête positivement] Est-ce que c'est honteux ? Voilà !

\section{Annexe 3 :}

Tableau récapitulatif des éléments non verbaux effectués par Marine Le Pen, en lien avec le contexte de filmage et la situation d'énonciation (20I2). Copyright : Morgane Belhadi. License : CC BY

\begin{tabular}{l} 
Descriptions \\
\hline Coupe, maquillage, tenues plus \\
modernes, vêtements amples, allure \\
imposante, chaîne ornée d'une croix
\end{tabular}

Faux sourires

Verre d'eau, lecture de journaux

Poings serrés côte à côte

Réaction de Jean-Luc Mélenchon : regard fixe et sourcils froncés; puis narines dilatées, regard hautain et lèvres pincées regard, mouvement de balancier du buste, «manipulatoires » (action de se gratter le nez, de passer la main dans ses cheveux)

Croiser les bras

\section{Significations}

Changement de « look» depuis 2004 : conquête du pouvoir, séduction, modernisation, avec des éléments de continuité de l'idéologie frontiste.

Ironie

Stratégie de l'évitement, montrer sa position de force dans les sondages, conquête du pouvoir (cf. changement de «look»)

Clignement d'yeux, détournement du Gêne (face à Jean-Luc Mélenchon)

Auto-défense, refus de débattre mais aussi position de repos car action effectuée sur un temps long

Geste asynchronique par rapport au terme "leurre ", ou bien mise en confrontation de deux concepts

Colère et détermination; puis colère et mépris 


\begin{tabular}{ll}
\hline Descriptions & Significations \\
\hline Filmage : split screens, zoom sur les & Renforcement de la dramatisation du \\
visages ; quasi gros plan de Marine & face-à-face, mise en avant des réac- \\
Le Pen et illusion d'optique (Marine & tions de chacun. Filmage en faveur \\
Le Pen semblant « dominer » David & de Marine Le Pen \\
Pujadas et Jean-Luc Mélenchon) & \\
\hline
\end{tabular}

\section{Annexe 4 : Transcription verbale et non verbale de l'extrait étudié (2012)}

DP (David Pujadas) Bonsoir, Jean-Luc Mélenchon.

JLM (Jean-Luc Mélenchon) Bonsoir.

DP Soyez le bienvenu.

JLM Bonsoir, madame. [Il s'assoit et regarde MLP]

MLP (Marine Le Pen) Bonsoir Monsieur [regard et sourire ironique furtifs vers JLM avant de le détourner pour prendre son verre d'eau]

DP Vous avez 20 minutes pour débattre. C'est vous qui commencez, Jean-Luc Mélenchon.

JLM Oui ... Tout à l'heure, madame Le Pen, vous vous êtes donné à bon compte le beau rôle à propos de l'égalité entre hommes et femmes. Et ce qui nous séparera, nos deux courants, ... depuis toujours et pour longtemps, ... c'est que nous nous sentons, nous, les militants de l'égalité humaine par-delà toutes les différences. Et tout à l'heure, au hasard d'une question, vous déclarez euh : "Oui, je suis pour l'égalité entre les hommes et les femmes. " Mais, madame, la première égalité, et vous le savez, c'est de pouvoir disposer librement de soi, de son corps. Or, vous [doigt accusateur/beat + deictic], vous êtes pour [doigt accusateur/beat + deictic] supprimer le remboursement [doigt accusateur/beat + deictic] par la Sécurité sociale de l'interruption volontaire de grossesse. Ce qui signifie que ce droit [MLP prend son verre d'eau], qu'aujourd'hui vous ne combattez plus - il n'y a plus un seul bulletin de vote dans notre pays contre le droit à l'avortement. Mais vous [doigt accusateur sur MLP, MLP avale une gorgée tout en reposant son verre, visage tourné vers DP] voulez le réserver seulement à celles qui en ont les moyens [elle pose son index et son pouce sur son menton, tête à moitié tournée vers JLM] Je dénonce cette hypocrisie [même posture de MLP, tête et regard baissés]. Et... 
DP Votre réponse, Marine Le Pen.

MLP [regard vers DP] D'abord, monsieur Pujadas, j'aimerais quand même vous dire quelque chose, parce que c'est un peu facile. [elle prend son inspiration, visage en direction de JLM mais regard baissé] Je voudrais vous dire que les...

JLM Ce n'est pas à moi que vous voulez parler ?

MLP ... Français attendent un débat serein et constructif lors cette élection présidentielle... [regard baissé] parce que ... les enjeux qui sont ceux de notre pays sont extrêmement importants. Or, ... en organisant ce débat, vous ne répondez pas aux attentes des Français [regard dans le vide]. Vous ne les ... entendez pas, d'ailleurs on se pose la question de savoir s'ils vous intéressent - à part peut-être pour l'audimat qu'ils apportent [regard vers DP]. Parce que [regard à nouveau baissé], on voit bien que ce débat a été organisé uniquement pour euh ... une logique commerciale. Ooon, vous êtes un peu le Paul Amar des temps modernes, hein [regard vers DP]. Vous auriez pu nous donner peut-être [elle remet une mèche sur le côté] une euh paire de gants de boxe, d'ailleurs [main dirigée vers JLM, regard toujours vers DP] Monsieur ... Mélenchon a tout de suite parlé d'un combat de boxe, de me traîner, je crois, d'un bout à l'autre du ring, avec ses dents peut-être ? [mouvement de balancier, sourcils froncés] Et vous êtes tombé dans ce, cette politique-spectacle, dans cette politique, euh [regard de face, dans le vide]...

JLM Vous ne voulez pas me répondre?

MLP ...commerciale, ...

JLM Vous allez faire durer ça, combien de temps?

MLP ... qui est

JLM Hé ! je suis là. [rires sur le plateau, plan sur JLM]

MLP ... profondément ...

DP Attendez, Jean-Luc Mélenchon.

MLP Monsieur Pujadas

JLM Quand vous tombez sur un contradicteur qui n'est pas complaisant, [MLP Profondam...] c'est fini. Hein ? Vous n'êtes plus là, vous.

MLP ... profondément, heu 
DP Attendez, Jean-Luc Mélenchon. Laissez parlez Marine Le Pen, puis moi, je dirai également un mot.

JLM D'accord.

MLP ...profondément déshonorant. Parce qu'on est aussi sur le service public, euh, et que imposer un adversaire à quelqu'un qu'on invite dans une émission, ça ne s'est jamais vu, ça ne s'est jamais fait, contrairement à ce que vous avez pu dire, vous ou votre patron [regards alternativement vers DP ou de face, dans le vide]. Euh, et, euh, je trouve ça, euh, je trouve qu'une fois de plus, c'est un ... une méthode qui m'est euh réservée. On sait comment s'est passé [regard tourné vers la droite, dans le vide, tout en passant sa main dans les cheveux] le premier «Des paroles et des actes ", d'ailleurs là aussi, il y a eu une méthode qui m'a été réservée, après on a changé, euh, le show.

$[\ldots]$

JLM [...] Cette dame [MLP] [...] a décidé que les femmes qui resteraient à la maison auraient droit à $80 \%$ du SMIC. Combien ça fait, monsieur Pujadas ? Ça fait 878 euros. [...] Autrement dit, cette dame a inventé quelque chose qui, sous prétexte de renvoyer les femmes à la maison ce à quoi nous sommes totalement opposés -, en plus les arnaque. Et vous l'avez entendu tout au long de la campagne : sitôt qu'elle tombe sur quelqu'un qui est en état de lui résister, de lui montrer que ce qu'elle raconte, ce ne sont que des fumées dangereuses, stimulatrices de haine, alors elle joue avec ses papiers comme elle est en train de le faire en ce moment, elle regarde ailleurs parce qu'elle a peur - et elle a bien raison.

[Monologue de JLM au cours duquel il critique la position du FN sur les étrangers, les femmes et la proposition de créer un salaire parental, mouvements de caméras alternativement sur lui et sur MLP qui lit ostensiblement le journal puis fait mine de rassembler ses notes]

DP On va demander à Marine Le Pen...

JLM Vous ne voulez toujours par répondre?

DP ... si elle souhaite tout de même s'exprimer sur le salaire parental, cette proposition dont vous dites qu'elle ne verrait le jour que si elle était possible financièrement. Donc, est-ce que c'est pour vous un moyen d'émanciper les femmes, de reconnaître ce travail à domicile, ou au contraire est-ce qu'il n'y a pas un risque d'enfermement dans le rôle de mère de famille? 
MLP Monsieur Pujadas, je vous ai exprimé - mais s'il faut que je le dise cinq fois, je le dirai cinq fois - que je n'entendais pas débattre avec Monsieur Mélenchon...

JLM Me reste-t-il du temps monsieur Pujadas ? [rires dans l'assistance] Pour vous faire de nouvelles révélations quant au programme...

DP Là il en reste un peu à Marine Le Pen.

MLP ... car il est, Monsieur Pujadas, un leurre. J'veux dire, quel est l'intérêt...

JLM Si j'étais un leurre, vous n'auriez pas si peur.

MLP Je vais vous dire une chose : on s'est rendu compte qu'il y avait d'autres leurres, d'ailleurs, dans cette campagne présidentielle. Il y a des leurres qui abandonnent avant, hein, c'est le cas de tous les candidats suscités par Nicolas Sarkozy et qui euh, sur ordre euh, abandonnent leur candidature parce que Nicolas Sarkozy est en danger, et donc euh, ils l'abandonnent à la demande de monsieur Sarkozy. C'est monsieur Borloo euh, c'est monsieur Nihous euh, c'est madame euh euh Boutin, c'est monsieur Morin, bon, euh, ce sont... [geste imprécis des mains]

JLM Monsieur Sarkozy lui-même, qui est d'accord avec vous sur l'essentiel.

MLP C'est ce qu'on appelle des leurres. Et puis, il y a les leurres qui sont censés servir à à à tromper une partie de l'électorat pour rapporter docilement en réalité au candidat euh au au second tour les voix. Et ça, c... c'est le rôle de monsieur Mélenchon. Or euh, je ne peux pas discuter avec un leurre.

JLM Mais discutez avec monsieur Pujadas ! Répondez-lui, madame, allons !

MLP Je ne peux discuter qu'avec un vrai candidat, quelqu'un qui a un vrai projet.

\section{Bibliographie}

Abélès, Marc (2007). Le spectacle du pouvoir. Paris : L'Herne, coll. Carnets de l'Herne.

McNeill, David (I992). Hand and Mind: What Gestures Reveal about Thought. Chicago, Londres : The University of Chicago Press 
Bonicco, Céline (2007). "Goffman et l'ordre de l'interaction : un exemple de sociologie compréhensive ", Philonsorbonne [En ligne] http://philon sorbonne.revues.org/IO2 Consulté le 3O.I I.I7

Burger, Marcel, Jacquin, Jérôme \& Micheli, Raphaël (eds) (20I I). La parole politique en confrontation dans les medias. Bruxelles : De Boeck.

Calbris, Geneviève (2003). L'expression gestuelle de la pensée d'un homme politique. Paris : CNRS Éditions.

Charaudeau, Patrick (2002). "A quoi sert d'analyse le discours politique ? ", in Análisi del discurs politic, Barcelone : IULA-UPF.

Charaudeau, Patrick (I995). "Ce que communiquer veut dire ", in Revue des Sciences humaines, $5 \mathrm{I}$.

Charaudeau, Patrick (I993). "Le contrat de communication dans la situation classe ", in J.-F. Halte et al., Interactions: actualité de la recherche et enjeux didactiques. Paris : CRELEF J.F.

Cosnier, Jacques \& Brossard, Alain (eds) (I984). La communication non verbale. Neuchâtel, Paris : Delachaux et Niestlé, coll. Textes de base en psychologie.

Coulomb-Gully, Marlène (200I). La démocratie mise en scènes. Télévision et élections. CNRS Communication.

Constantin de Chanay, Hugues \& Kerbrat-Orecchioni, Catherine (2017) « Regard et deixis personnelle : l'adresse dans les débats d'entre-deux-tours des élections présidentielles françaises ", Langue française I93, 93-108.

Constantin de Chanay, Hugues \& Turbide, Olivier, " Les discours politiques. Approches interactionnistes et multimodales » (20I I). Mots. Les langages $d u$ politique 96 [En ligne] http://mots.revues.org/20I70 Consulté le 02.I2.I7.

Esquenazi, Jean-Pierre (I999). Télévision et démocratie. La politique à la télévision française, 1958-1990. Paris: Presses universitaires de France.

Ekman, Paul \& Boucher, Jerry ID. (I975). "Facial Areas and Emotional Information ", Journal of Communication 25, $\mathrm{n}^{\circ} 5$, pp. 2 I-29. http://onlinelibrary.wiley.com.ezproxy.univ-paris3.fr/doi/IO. I I I I/j. I 4602466. I 975 . tboo577.x/pdf Consulté le 06.03.20I6.

Ekman, Paul (I976). "Movements with Precise Meanings", Journal of Communication 26, 3, I4-26. http://onlinelibrary.wiley.com.ezproxy.univparis3.fr/doi/IO.I I I I/j.I4602466.I976.tbor898.x/pdf Consulté le o6.03. 2016.

Goffman, Erving (1973). La mise en scène de la vie quotidienne. I. La présentation de soi. Paris : coll. Le sens commun. Les Éditions de Minuit. 
Jacquin, Jérôme (20II). "Orientation interactionnelle et multimodale vers l'activité de 'débattre'. Analyse d'un extrait de débat public ", Mots. Les langages du politique 96 http://mots.revues.org/20336 Consulté le 03.I2.I7.

Kerbrat-Orecchioni, Catherine (2013). "Humour et ironie dans le débat Hollande-Sarkozy de l'entre-deux-tours des élections présidentielles ( 2 mai 20I 2) ", Langage et société I 46, 49-69.

Mery Marwan (2014). Vous mentez! Détecter le mensonge, démasquer les menteurs, Paris : Eyrolles

Pasquier, Sylvain (2008). "Le corps chez Goffman ", in Revue du Mauss permanente.

Poggi, Isabella, D’Errico, Francesca \& Vincze, Laura (20II). "Discrediting moves in political debates ", in Second International Workshop on User Models for Motivational Systems: the affective and the rational routes to persuasion. Girona, Spain: Springer LNCS, 84-99.

Winkin, Yves (1984). La nouvelle communication. Paris : Seuil, coll. PointsEssais.

\section{Vidéographie}

Extrait de l'émission Mots croisés, France 2, 27.05.2002, durée : 2h. Consulté sur place le 20.0I.I 5 aux archives de l'Institut National de l'Audiovisuel (InaTHEQUE, Bibliothèque nationale de France, Paris)

Extrait de l'émission Des paroles et des actes, France 2, 23.02.20I2, durée : 2 h 5I, durée du duel Marine Le Pen/Jean-Luc Mélenchon : $24 \mathrm{mn}$, durée du premier extrait : « $3 \mathrm{mn}$ 02, durée du second extrait : $7 \mathrm{mn}$ I 8 . https://www. youtube.com/watch?v=wsxnmPa39yU Consulté le 03.I2.I8. 